\title{
Imaging of polar cap patches with a low-cost airglow camera: pilot observations in Svalbard, Norway
}

\author{
Keisuke Hosokawa ${ }^{1^{*}}$ (D) Yasunobu Ogawa ${ }^{2}$ and Satoshi Taguchi ${ }^{3}$
}

\begin{abstract}
We evaluate the capability of a low-cost all-sky imager (ASI), which has been operative in Longyearbyen $\left(78.1^{\circ} \mathrm{N}, 15.5^{\circ}\right.$ E), Norway, to detect $630.0 \mathrm{~nm}$ airglow signatures of polar cap patches. The ASI is composed of a small camera, with a charge-coupled device (CCD), manufactured by Watec Co. Ltd., a fish-eye lens, and an optical filter whose central wavelength is $632.0 \mathrm{~nm}$ and full-width half maximum (FWHM) is $10 \mathrm{~nm}$. In Longyearbyen, another ASI equipped with a cooled electron-multiplying charge-coupled device (EMCCD) camera has been operative for observations of polar cap patches. We compare the images from the two systems and investigate the performance of the low-cost ASI. On the night of December 4, 2013, a series of polar cap patches were observed by the EMCCD ASI. The low-cost ASI also detected regions of enhanced $630.0 \mathrm{~nm}$ airglow passing through the fields-of-view. The quality of the raw images from the low-cost ASI obtained every $4 \mathrm{~s}$ were visibly much worse than that of the EMCCD ASI. However, an integration of 7-15 consecutive images made it possible to capture the temporal evolution and spatial structure of the patches, for example, their anti-sunward propagation and finger-like structures along the trailing edge. The estimated values of the absolute optical intensity from the low-cost ASI were found to be consistent with those from the EMCCD ASI, whereby the offset was $<100$ R. This offset can be explained by the contribution of the background continuum emission to the low-cost ASI images, because the band width of the optical filter used for the low-cost ASI is $\sim 3$ times wider than that used for the EMCCD ASI. The results indicate that the airglow measurement with the low-cost ASI is feasible even for quantitative studies of F-region phenomena such as the dynamics of polar cap patches.
\end{abstract}

Keywords: Ionosphere, Polar cap patches, Airglow, Optical observations, Satellite navigation

\section{Introduction}

During the night, weak and almost invisible light, referred to as airglow or nightglow, is released from the Earth's upper atmosphere. There are two major emission lines of airglow from atomic oxygen; at $557.7 \mathrm{~nm}$ and $630.0 \mathrm{~nm}$. Both of these have been used to study various phenomena in the mesosphere and thermosphere. The optical intensity of the $630.0 \mathrm{~nm}$ emission of oxygen (so-called red-line oxygen emission) from the lower thermosphere at an altitude of $200-300 \mathrm{~km}$ is known to be proportional to the densities of $\mathrm{O}^{+}$and $\mathrm{O}_{2}$ (e.g., Sobral et al.

\footnotetext{
*Correspondence: keisuke.hosokawa@uec.ac.jp

${ }^{1}$ University of Electro-Communications, Chofugaoka 1-5-1, Chofu, Tokyo 182-8585, Japan

Full list of author information is available at the end of the article
}

1993). The $630.0 \mathrm{~nm}$ emission can, therefore, be used as a proxy for variations in the electron density slightly below the peak height of the F-region ionosphere. In the last 2 decades, the oxygen red-line emission observed with all-sky airglow imagers (ASIs) equipped with cooled charge-coupled device (CCD) cameras has been widely used to observe various kinds of ionospheric phenomena (Shiokawa et al. 2009, and references therein). High signal-to-noise ratio images of the ionosphere from ASIs have enabled us to observe the detailed two-dimensional structure and temporal evolution of plasma bubbles (e.g., Otsuka et al. 2002) in the low-latitude region, as well as traveling ionospheric disturbances of various scales in the mid-latitude region (Shiokawa et al. 2003a, b).

In the last decade, similar airglow observations at highlatitudes have made it possible to visualize the dynamic 
behavior of polar cap patches (Hosokawa et al. 2006; Moen et al. 2007). Polar cap patches are known as regions of high-density plasma in the F-region, which are generated near the dayside cusp and subsequently propagate towards the nightside across the central polar cap (Crowley 1996, and references therein). The dynamic characteristics and generation processes of polar cap patches have been investigated for almost 3 decades since their discovery in early 1980s (Weber et al. 1984). In recent years, it has been commonly recognized that the twodimensional imaging capability of ASIs is useful for visualizing the large-scale spatial structure of polar cap patches (Hosokawa et al. 2014, and references therein). For instance, Hosokawa et al. (2009b) demonstrated that the overall motion of patches is controlled by the magnitude and orientation of the interplanetary magnetic field (IMF). Hosokawa et al. (2009a) combined observations of plasma irregularities by the coherent HF radar of SuperDARN using ASI data from Resolute Bay, Canada, and showed that plasma irregularities are distributed in the entire region of the patches. More recently, Hosokawa et al. (2013a, b) identified mesoscale structures within or in the vicinity of polar cap patches by utilizing the high spatial and temporal resolution of the electron-multiplying charge-coupled device (EMCCD) ASI system in Longyearbyen, Norway.

As mentioned previously, ASIs are one of the essential tools for monitoring the spatial and temporal evolution of various ionospheric phenomena at all latitude regions. However, it is still very difficult to make a dense network of ASIs and to monitor the large-scale structure of such ionospheric phenomena, because ASI systems are relatively expensive. In addition, conventional ASIs equipped with a cooled CCD detector tend to be large (e.g., Shiokawa 1999); thus, the engineering work at the time of deployment and during maintenance would be difficult, especially in the severe conditions of the high Arctic and Antarctic regions. If a low-cost, compact ASI can be developed with the confirmed capability of observing the $630.0 \mathrm{~nm}$ airglow, it would contribute to building a network of $630.0 \mathrm{~nm}$ airglow observations over a large area. Recently, Hosokawa et al. (2014) combined data from two ASIs in Resolute Bay and Longyearbyen, respectively, and demonstrated that the measurements of the $630.0 \mathrm{~nm}$ airglow taken with a large field-of-view (FOV) provide a powerful monitoring system for the propagation characteristics of polar cap patches. In this sense, there is a strong demand to construct a network of $630.0 \mathrm{~nm}$ airglow measurement using low-cost, compact ASI systems.

For this purpose, we developed an ASI system for observation of $630.0 \mathrm{~nm}$ airglow emission. The ASI is composed of a low-cost camera, fish-eye lens, and an optical filter. We have been operating this low-cost ASI in Longyearbyen, Norway, since October 2013 to evaluate its performance. In this paper, we investigate the ASI data obtained during an interval of polar cap patches on the night of December 4, 2013. In particular, we compare the data from the low-cost ASI with those from an EMCCD ASI operating at the same observatory. Through this comparison, we quantitatively evaluate the feasibility of making $630.0 \mathrm{~nm}$ airglow measurements with the lowcost ASI system.

\section{Instruments}

In this study, we employ two ASI systems that have been operative in Longyearbyen $\left(78.1^{\circ} \mathrm{N}, 15.5^{\circ} \mathrm{E}\right)$, Norway. The low-cost ASI (hereafter called WATEC ASI) consists of a small CCD camera (WATEC-910HX, manufactured by Watec Co. Ltd., Japan), a narrow pass-band optical filter (Edmund Co. Ltd., with a central wavelength of $632.0 \mathrm{~nm}$ and full-width half maximum (FWHM) of $10 \mathrm{~nm}$ ), and a fish-eye lens (YV2.2X1.4A-SA2, Fujinon Co. Ltd.). We accumulated 256 frames (the exposure for one single frame is $1 / 60 \mathrm{~s}$ ) before streaming from the camera as a video signal, and thus, the exposure time of the streaming images is $\sim 4.3 \mathrm{~s}$. These raw images from the WATEC ASI were digitized (8 bit: $0-255$ ) every $4 \mathrm{~s}$ with a video encoder (AXIS Q7404, AXIS Co. Ltd.) and recorded by a computer as $640 \times 480$ pixels JPEG files. There is an offset of $2 \mathrm{~nm}$ between the central wavelengths of the optical filter $(632.0 \mathrm{~nm})$ from the $630.0 \mathrm{~nm}$ airglow line. However, the filter provides good coverage of the $630.0 \mathrm{~nm}$ emission because of its wide bandwidth $(\sim 10 \mathrm{~nm})$. The WATEC ASI is operative when the sun is below the horizon, even during the full moon period. The technical details of the WATEC ASI system, including the photos of the ASI system, are fully detailed by Ogawa (2019). The cost of the entire WATEC system is $\sim 1000$ USD, which is approximately $1 \%$ of that of the large cooled CCD ASI system.

The other optical system used in this study is an ASI equipped with a cooled EMCCD detector (hereafter called EMCCD ASI), which is composed of a Nikon ASI-2 system (Taguchi et al. 2004) and an EMCCD camera (C9100-13, Hamamatsu Photonics), which has an imaging part of $512 \times 512$ pixels. Narrow passband interference filters (Andover Co. Ltd.) are used for observing oxygen emissions at $557.7 \mathrm{~nm}$ and $630.0 \mathrm{~nm}$. The background continuum emission is also sampled at a wavelength of $572.5 \mathrm{~nm}$ (where no distinct airglow line exists) to derive the absolute optical intensity. The imager was deployed in Longyearbyen, Norway, in October 2012 (Taguchi et al. 2012), and has since been operating without any technical problems. The EMCCD ASI observes the $630.0 \mathrm{~nm}$ airglow images with an exposure time of $4 \mathrm{~s}$ to detect weak airglow signatures of polar cap patches. 
Details of this ASI system are documented in Taguchi et al. (2012), and some recent examples of polar cap patch measurements can be found in Hosokawa et al. (2016a, b). In this study, we employ the $630.0 \mathrm{~nm}$ airglow images from the EMCCD ASI to evaluate the performance of the WATEC ASI.

We note that, at the time of comparison, the central wavelength of the optical filter for the EMCCD ASI is $630.0 \mathrm{~nm}$ and FWHM is $3 \mathrm{~nm}$, which is narrower than that of the optical filter used for the WATEC ASI, and as such may introduce a difference in the absolute optical intensity values from the two ASI systems. The WATEC imager cannot use the narrow pass-band filter that was used for the EMCCD imager. The reason is as follows: to prevent a decrease in transmittance, the WATEC imager does not produce parallel ray path in the optics. Therefore, the light passes the filter obliquely. As the incident angle increases, the transmission region shifts to the shorter wavelength, while the shape of the transmittance band does not change significantly. As described in Ogawa (2019), the incident angles of the ray path are mostly within about $18 \mathrm{deg}$. The use of relative wider pass-band filter allows us to observe the $630.0 \mathrm{~nm}$ emission even when the incident angle reaches this value (i.e., $\left.18^{\circ}\right)$.

\section{Observations}

Figure 1a shows the temporal variation of the IMF $B_{z}$, which was obtained by the ACE spacecraft located far upstream of the Earth's magnetosphere during a 4-h interval on December 4, 2013; the IMF $B_{z}$ was mostly negative during the interval. Several previous studies (e.g., Hosokawa et al. 2009a) showed that polar cap patches are often observed during intervals of continuous southward IMF, and thus, the background condition of the current interval is suitable for observations of patches on the nightside. Figure $1 \mathrm{~b}$ shows a time-series of the south-to-north cross-section of the $630.0 \mathrm{~nm}$ images (so-called keogram) from the EMCCD ASI. The central time of this interval roughly corresponds to the magnetic midnight (i.e., $24 \mathrm{MLT}$ ). The data have already been converted to the absolute optical intensity in units of

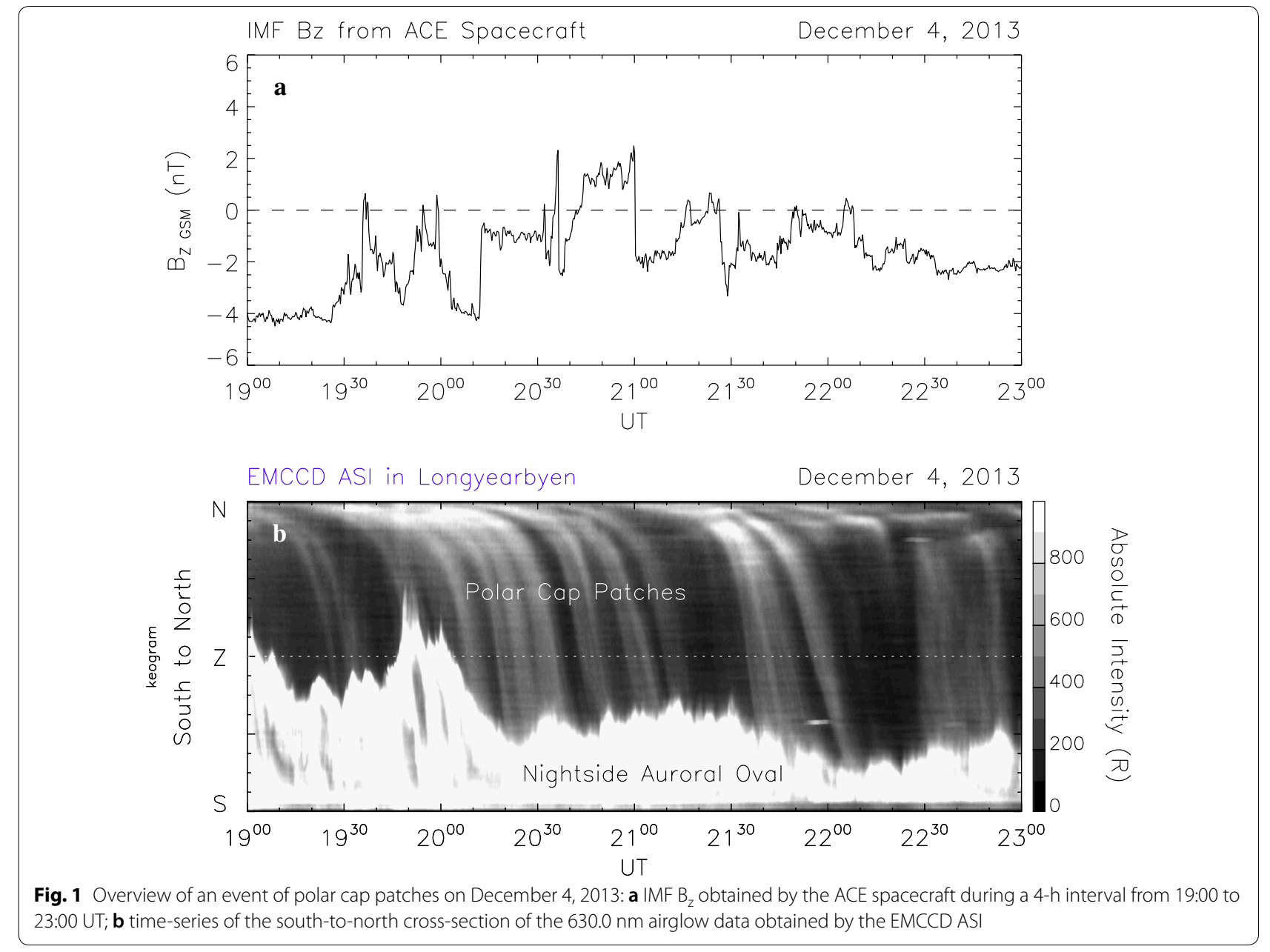


Rayleigh (R) using methods described by Shiokawa et al. (2000). The continuous bright structure seen in the equatorward half of the FOV is the poleward part of the auroral oval on the nightside. Several faint traces, which can be seen passing through the zenith and eventually diving into the auroral oval, are signatures of polar cap patches in the airglow observations. We observed a total of 10 patches during the 4-h interval, and their peak brightness at zenith was 350-450 R.

Here, we directly compare the $630.0 \mathrm{~nm}$ images obtained from the WATEC and EMCCD ASIs during the interval of the polar cap patches, as shown in Fig. 1. Figure 2b shows a $630.0 \mathrm{~nm}$ all-sky image from the EMCCD ASI at around 20:35 UT, near to the middle of the 4-h interval shown in Fig. 1. The equatorward half of the FOV is illuminated by the bright aurora appeared in the south of Longyearbyen. In the central part of the FOV, however, a faint airglow structure was captured, which was a manifestation of a polar cap patch. This patch was drifting from north to south (from top to bottom in Fig. 2b) and this corresponds to the general equatorward motion of polar cap patches on the nightside (Hosokawa et al. 2009b). As shown by Hosokawa et al. (2013b), the EMCCD ASI is able to visualize the detailed spatial structure of polar cap patches with high spatial resolution; for example, there were a few finger-like structures along the trailing edge of the patch. Hosokawa et al. (2016b) used data from the EMCCD ASI to demonstrate that such features are only seen along the trailing edge of patches (i.e., not along the leading edge of patches), and that their generation can be explained by plasma structuring through the gradient-drift instability (Keskinen and Ossakow 1983).

Figure 2a shows a raw $630.0 \mathrm{~nm}$ all-sky image simultaneously obtained by the WATEC ASI with an exposure time of $4 \mathrm{~s}$. Although the rough outline of the patch can be identified, it is almost impossible to visualize the detailed spatial structure, for example, the finger-like structure seen in Fig. 2b. To improve the signal-to-noise ratio of the image, we integrated consecutive images and derived average images. Figure $2 \mathrm{c}$ shows an average of seven consecutive images, the total exposure time being $\sim 30 \mathrm{~s}$. The reduction of the noise makes it possible to see the large-scale structure of the patch. Figure $2 d$ shows an average of 15 images, thereby making it possible to see slight indications of the finger-like structure along the trailing edge. Ogawa (2019) estimated the average noise level of the raw images from the $630.0 \mathrm{~nm}$ WATEC ASI to be $30 \mathrm{R}$. Because thermal noise is known to be inversely proportional to the square root of the number of images for integration, the noise in Fig. 2c would be reduced to $\sim 1 / 4$ of that in Fig. $2 \mathrm{a}$, which is $\sim 8$ $\mathrm{R}$. The typical luminosity of polar cap patches is $100-300$
R (e.g., Hosokawa et al. 2009b), and thus, the signal-tonoise ratio from the WATEC ASI would range from 12 to 37 when we employ 15 images for averaging.

Figure $2 \mathrm{e}$ and $\mathrm{f}$ shows averages of 30 and 75 images, respectively. The signal-to-noise ratio is further improved, but the structure of the patch is somewhat blurred. This is primarily because patches normally move with a background convection speed of $500-1000 \mathrm{~m} \mathrm{~s}^{-1}$ (Hosokawa et al. 2009b). Hence, there is a trade-off between the signal-to-noise ratio of the images and the discreteness of the observed patch structure, which makes it difficult to select the best integration time in the post-processing procedure. During the current interval, however, the averages of 7 or 15 images (i.e., $30 \mathrm{~s}$ or $1 \mathrm{~min}$ total integration times) would be appropriate for visualizing the spatial structure of the patch in various scale sizes. An animation of the all-sky images from the simultaneous measurements of EMCCD and WATEC ASIs is provided as Additional file 1. The animation better demonstrates that the quality of the WATEC ASI data is sufficient for visualizing the propagation characteristics and large-scale spatial structure of polar cap patches.

The WATEC ASI data shown in Fig. 2 are raw count images that cannot be used for quantitative analysis. Here, we try to derive the absolute optical intensity for the WATEC ASI images. Figure 3a again shows an average of fifteen consecutive raw images obtained by the WATEC ASI at around 20:35 UT. The faint structure of the patch is seen in the middle of the FOV. Figure $3 \mathrm{~b}$ gives a distribution of the sensitivity values in units of raw count $R^{-1} s^{-1}$, which have been calculated using data taken during a calibration using a 76-inch integrating sphere (Labsphere LMS-760) at the National Institute of Polar Research, (NIPR), Japan. The sensitivity values are not uniform within the FOV, but the sensitivity is 0.02 Count $\mathrm{R}^{-1} \mathrm{~s}^{-1}$ near the central part of the FOV. Since the raw images are digitized with 8 bits, the maximum raw count is 255 . When we consider the sensitivity of 0.02 Count $\mathrm{R}^{-1} \mathrm{~s}^{-1}$ and the exposure time of $\sim 4.3 \mathrm{~s}$, the maximum raw count of 255 corresponds to $2964 R$ $(=255 / 0.02 / 4.3)$ with the current setting. Figure $3 \mathrm{c}$ shows the absolute optical intensity image derived from the raw image in Fig. 3a. When deriving the absolute optical intensity, first, we estimate the dark count using pixels outside the FOV of the ASI and subtract it from the raw count values. After the subtraction, we convert the raw count values to the absolute optical intensity in units of Rayleigh using the sensitivity values shown in Fig. 3b. In the following, we compare these calibrated data with those from the EMCCD ASI, which was also calibrated using the same integrating sphere at NIPR.

Figure 4a shows the EMCCD ASI data in a format of keogram for a 3-h interval from 20:00 to 23:00 UT 


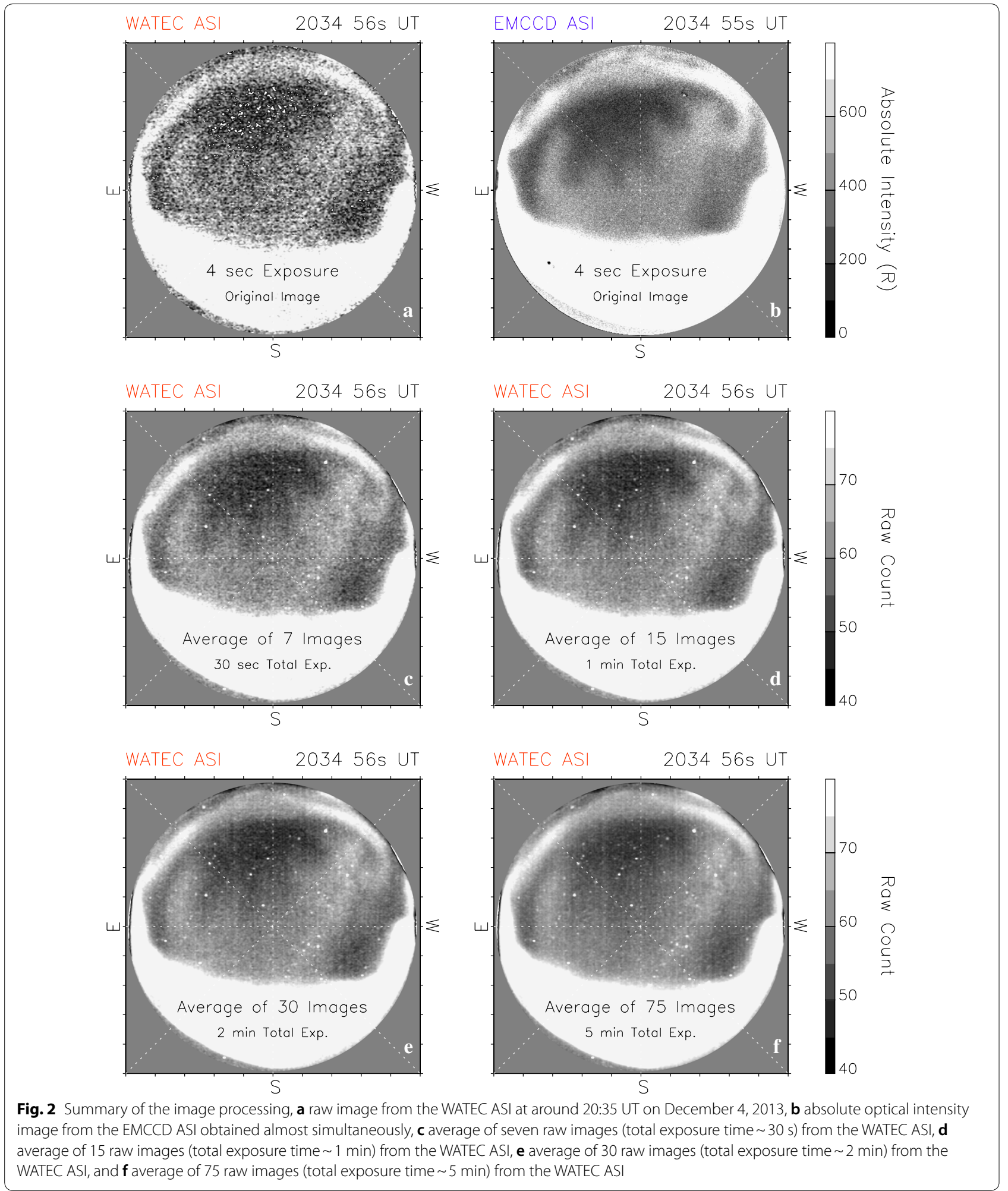

on December 4, 2013. Faint traces of polar cap patches are again seen to propagate across the FOV of the ASI. In Fig. $4 \mathrm{~b}$, the absolute optical intensity data from the simultaneous WATEC ASI measurement are shown in the same format, which indicates that the WATEC ASI data were able to capture the anti-sunward propagation 

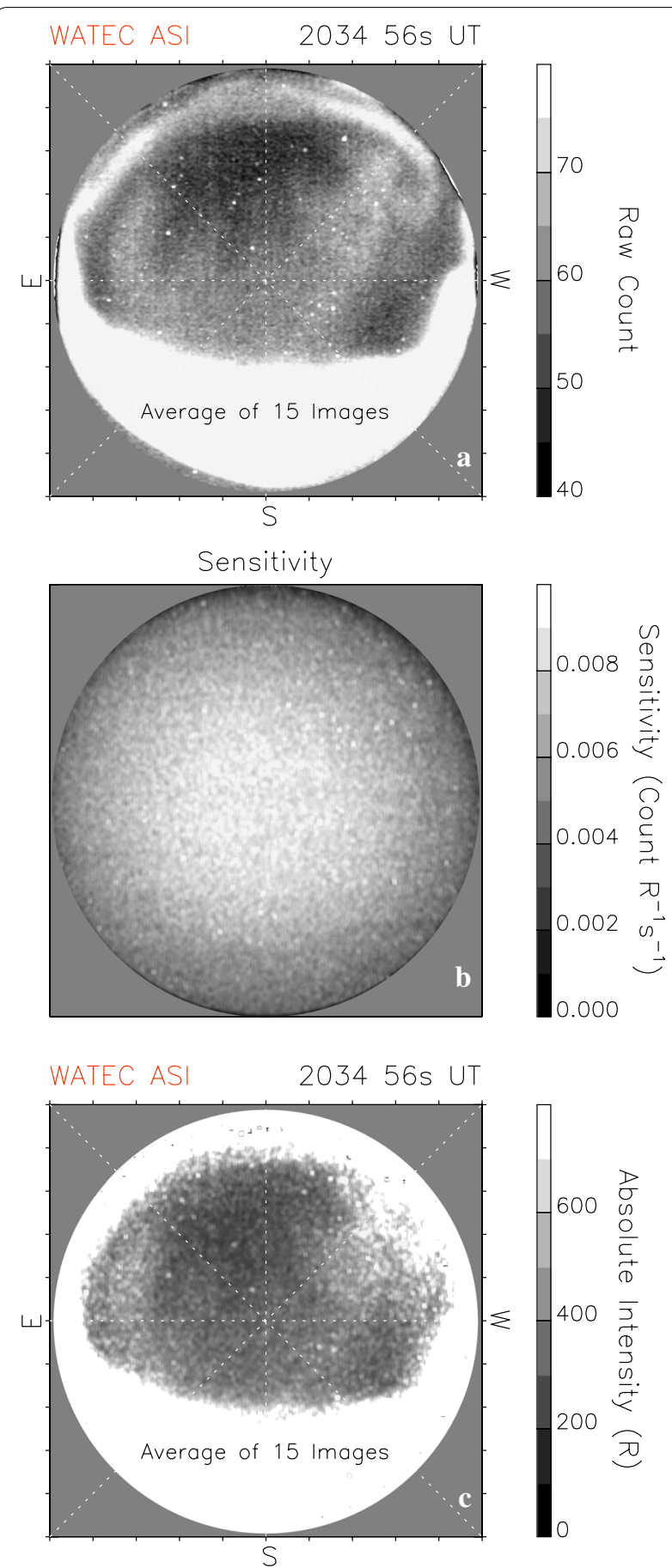

Fig. 3 Summary of the calibration process, a raw image from the WATEC ASI at around 20:35 UT on December 4, 2013, b sensitivity distribution of the WATEC ASI derived from a calibration using integrating sphere, and $\mathbf{c}$ absolute optical intensity image of the WATEC ASI at around 20:35 UT

of the patches. Figure $4 \mathrm{c}$ shows a time-series of the zenith intensity of the EMCCD (blue) and WATEC (red) ASIs. In both data sets, there are many enhancements associated with the passage of the polar cap patches across the zenith. The time-series from the two ASI systems vary in tandem. However, there is a slight offset between the data from the two systems especially when the optical intensity is low (e.g., 21:15 to 21:30 UT, and 22:05 to 22:25 UT). The difference is as large as $100 \mathrm{R}$; this difference is considered in the discussion.

\section{Discussion}

In the previous section, we showed that the data from the two ASI systems are generally consistent with each other if we average the images from the WATEC ASI and improve the signal-to-noise ratio in the post-processing process. Figure 5 shows the correlation between the absolute optical intensity values from the two ASI systems, which again indicates that the quality of the data from the WATEC ASI is sufficient for visualizing the temporal evolution of polar cap patches. However, in the second half of the interval, especially when the airglow intensity is low, there is an offset of $\sim 50$ to $100 \mathrm{R}$ between the absolute optical intensity values from the two systems. To discuss the potential source of this difference, the difference in the characteristics of the optical filters that were used in the two systems should be taken into account. Figure 6 shows the characteristics of the optical filters used for the EMCCD (blue) and WATEC (red) ASIs, which was obtained by a grating spectrometer at the NIPR. Both the filters provide good coverage of the $630.0 \mathrm{~nm}$ airglow line, but the FWHM of the WATEC ASI filter is $\sim 10 \mathrm{~nm}$, whereas the EMCCD ASI filter is only $3 \mathrm{~nm}$. The wider coverage of the filter introduces a larger contribution of the background continuum emission to the WATEC ASI data, which is the primary reason for the offset seen in Fig. 4c. In Fig. 6, we also plot the airglow spectrum that was obtained at the same time by the Auroral Spectrograph (ASG) operating in Longyearbyen (Taguchi et al. 2002). There exists an outstanding peak at $630.0 \mathrm{~nm}$, which is the primary target of our airglow measurements. We can identify another peak at $636.4 \mathrm{~nm}$, which is also an oxygen emission from the lower thermosphere. The wider optical filter for the WATEC ASI slightly overlaps the airglow line at $636.4 \mathrm{~nm}$; thus, the contribution of this additional oxygen emission might have contributed to the offset. However, if the contribution of background continuum emission and the additional airglow line at $636.4 \mathrm{~nm}$ is the only reason for the difference, the offset between the two ASI systems should be stable throughout the interval. In particular, there should not be any differences in the offset between the intervals of airglow patches and no patches. This is actually not the case in Fig. 4c. That is, the offset is not systematic; for example, the EMCCD ASI gives higher values at around 2030 UT, while the WATEC ASI gives higher values around 2130 

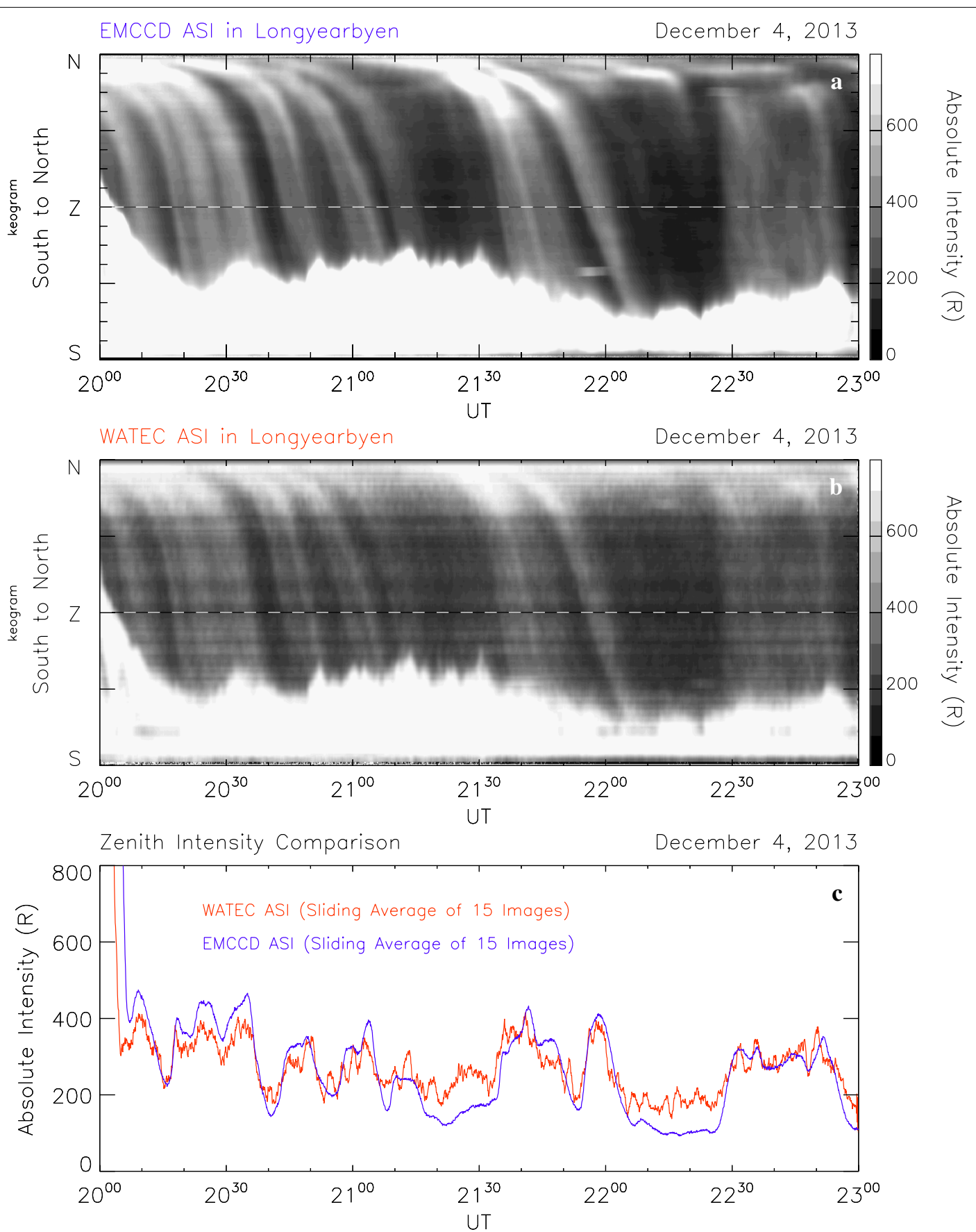

Fig. 4 Comparison of the WATEC ASI and EMCCD ASI, a time-series of the south-to-north cross-section of the absolute optical intensity of $630.0 \mathrm{~nm}$ airglow from the EMCCD ASI for a 3-h interval from 20:00 to 23:00 UT on December 4, 2013, b time-series of the south-to-north cross-section of the absolute optical intensity of $630.0 \mathrm{~nm}$ airglow from the WATEC ASI, and c time-series of the zenith intensity from the EMCCD (blue) and WATEC (red) ASIs

UT. We have not yet reached any definitive conclusion on what factors introduced this difference.

In this paper, we have shown that the low-cost, compact airglow imager can be used for the study of polar cap patches. The utilization of such an ASI system would contribute to the expansion of the existing network of ASIs in the polar cap regions. Currently, several imagers have been operative at polar cap latitudes, for example, at Longyearbyen and Ny Alesund in Norway, Resolute Bay and Eureka in Canada, and a few sites in northern 


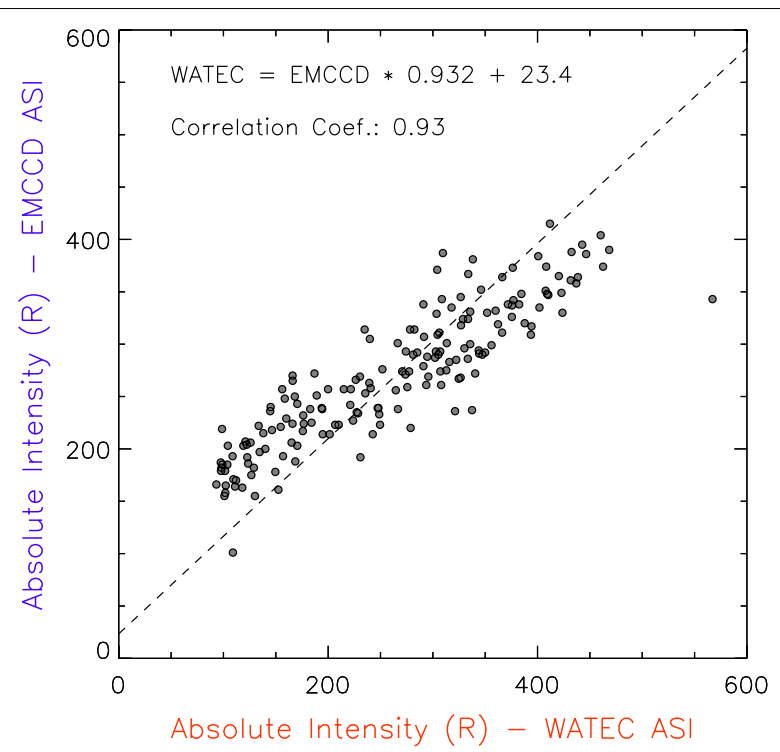

Fig. 5 Relationship between the absolute optical intensity values at zenith from the two ASI systems during the interval of polar cap patches shown in Fig. 4. Note that we only used data from 2010 to 2300 UT to remove the effect of large increase of emission intensity due to the appearance of aurora near the zenith in the first 10 min of the interval

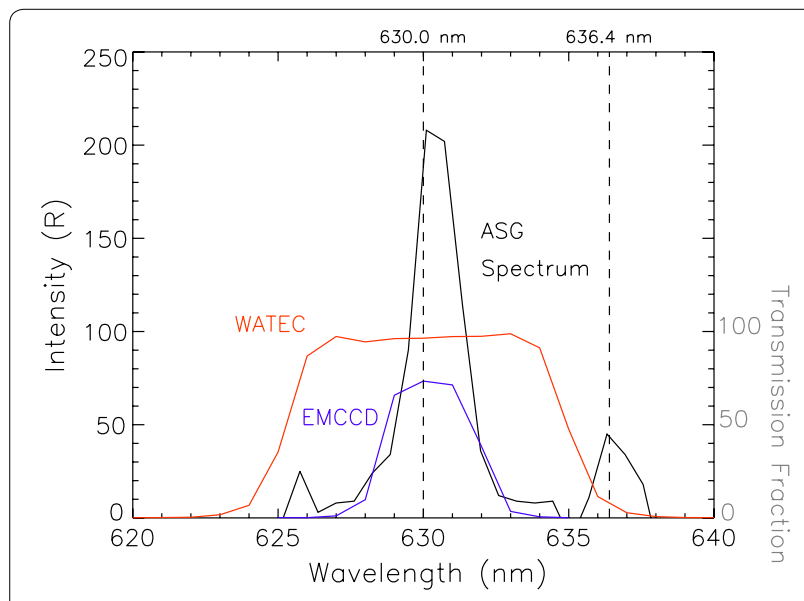

Fig. 6 Filter characteristics of EMCCD (blue) and WATEC (red) ASIs. The black curve shows the airglow spectrum obtained by the Auroral Spectrograph (ASG) operating in Svalbard at the same time

Greenland. To date, however, we have not been able to perform global imaging of the polar cap ionosphere by combining these ASIs, because the coverage of their FOV is still limited. If we deploy the WATEC ASI systems at several sites in northern Canada, Alaska, and Greenland, then a network of $630.0 \mathrm{~nm}$ airglow measurements with a much larger coverage would be established. In the southern hemisphere, there are a few on-going projects of unmanned networks of Geospace monitoring systems, which will be installed in the inland region of Antarctica (Ogawa 2019, and references therein). Although it is necessary to further reduce power consumption and establish a data transfer procedure in this location, if we install the WATEC ASI in such an unmanned observatory, a network of $630.0 \mathrm{~nm}$ airglow observations would be possible even at the polar cap latitudes in the southern hemisphere. This network would be used to carry out the interhemispheric comparison study of the polar cap patch and arc characteristics.

Another possible use of the WATEC ASI is for airglow measurements at mid-latitudes, low-latitudes, and in the equatorial regions. In these regions, various phenomena occur in the F-region ionosphere during nighttime, such as plasma bubbles and MSTIDs. In earlier literature, larger ASI systems such as OMTIs (Shiokawa et al. 2009) have been used for measuring such features. Kubota et al. (2000) carried out a campaign measurement of MSTIDs by distributing five large ASI systems in mainland Japan. They succeeded in visualizing the large-scale structure of southwestward propagating wave fronts of MSTID across the Japanese continent. However, the source and destination of such features are still unknown due to the limited coverage of ASIs in the mid-latitude region. In the case of plasma bubbles, most of the airglow measurements are regional. If an array of the WATEC ASIs is established in the southeast Asia and Oceania region, it would be possible to monitor the temporal evolution of a plasma bubble from its initiation. For such future measurements in the mid- and low-latitude regions, we have installed the WATEC ASIs into several test sites in mid- and low-latitude regions, and tried to evaluate their capability of detecting MSTIDs and plasma bubbles. Once the feasibility of such airglow measurements is confirmed, the WATEC ASI will open a new door for the global imaging of the ionosphere with ground-based airglow measurements.

\section{Summary}

We have employed a low-cost CCD camera (WAT910HX, Watec Co. Ltd) for airglow measurements in the polar regions. The camera is composed of a fish-eye lens and an optical filter whose central wavelength is $632.0 \mathrm{~nm}$, FWHM is $\sim 10 \mathrm{~nm}$, and transmittance is $85 \%$. The aim is to observe the $630.0 \mathrm{~nm}$ airglow from the F-region ionosphere in a two-dimensional fashion. The low-cost ASI was installed in Longyearbyen $\left(78.1^{\circ} \mathrm{N}\right.$, $15.5^{\circ} \mathrm{E}$ ), Norway, in October 2013 , and operated continuously during the winter of $2013 / 2014$. In this paper, we attempted to use the low-cost ASI to observe faint airglow variations caused by polar cap patches, which are regions of weak airglow enhancement in the polar cap regions. In Longyearbyen, airglow measurements with 
an ASI equipped with EMCCD detector (EMCCD ASI) have been carried out; thus, we are able to compare the images from these two ASI systems and evaluate the performance of the low-cost ASI quantitatively. On the night of December 4, 2013, a series of polar cap patches were observed by the EMCCD ASI. The optical intensity of the patches was as large as 400 Rayleigh. Simultaneous to this, the low-cost ASI also detected regions of enhanced airglow intensity passing through their FOV. The signalto-noise ratio of the raw images obtained every $4 \mathrm{~s}$ was evidently lower than those from the EMCCD ASI, but integration of 7-15 consecutive images made it possible to capture the two-dimensional structure of the polar cap patches. However, the estimated values of the absolute optical intensity from the low-cost ASI were found to be slightly larger than those from the EMCCD ASI when the optical intensity was low. This offset may be explained by the fact that the bandwidth of the optical filters used for the low-cost ASI is three times wider than that of the EMCCD ASI. The results of the current study demonstrate that the $630.0 \mathrm{~nm}$ airglow measurements using a low-cost and compact CCD camera can be used for studies of various phenomena in the F-region ionosphere.

\section{Supplementary information}

Supplementary information accompanies this paper at https://doi. org/10.1186/s40623-019-1094-7.

Additional file 1. Animation showing the temporal evolution of the polar cap patches between 1900 UT and 2300 UT on December 4, 2013 by the EMCCD ASI (left) and the WATEC ASI (right).

\section{Abbreviations}

ASI: all-sky imager; EMCCD: Electron Multiplying Charged-Coupled Device; FWHM: Full Width Half Maximum; FOV: field-of-view; IMF: interplanetary mag netic field; MSTIDs: medium-scale traveling ionospheric disturbances; OMTIs: optical mesosphere thermosphere imagers; SuperDARN: Super Dual Auroral Radar Network; UT: Universal Time.

\section{Acknowledgements}

We would like to thank Fred Sigernes for their help at UNIS, Longyearbyen. We also thank Shin Suzuki, Kazuo Shiokawa, and Akira Kadokura for their helpful comments for making the EMCCD ASI and its calibration, and Takeshi Aoki, and staff of Manufacturing Design Center for facilitating our work in the University of Electro-Communications.

\section{Authors' contributions}

$\mathrm{KH}$ designed and conducted the current research and prepared the manuscript. YO developed and calibrated the low-cost all-sky imager used for the observation. ST operated and maintained the EMCCD ASI in Longyearbyen and discussed the results of the data analysis. All authors read and approved the final manuscript.

\section{Funding}

This work was supported by JSPS-Kakenhi $(22340143,26302006$, and 17H02967).

\section{Availability of data and materials}

The EMCCD ASI data used in this paper can be provided on request from Prof. Satoshi Taguchi, who is the primary investigator of the optical observation project. The WATEC ASI data used in this paper can be provided on request from Prof. Yasunobu Ogawa. The solar wind data from the ACE spacecraft can be accessed through the ACE science center at http://www.srl.caltech.edu/ ACE/ASC/

\section{Ethics approval and consent to participate}

Not applicable.

\section{Consent for publication \\ Not applicable.}

\section{Competing interests}

The authors declare that they have no competing interests.

\section{Author details}

${ }^{1}$ University of Electro-Communications, Chofugaoka 1-5-1, Chofu, Tokyo 182-8585, Japan. ${ }^{2}$ Midoricho 10-3, Tachikawa, Tokyo 190-8518, Japan. ${ }^{3}$ Graduate School of Science, Kyoto University, Kitashirakawa-Oiwakecho, Sakyo-ku, Kyoto 606-8502, Japan.

Received: 12 July 2019 Accepted: 22 October 2019

Published online: 07 November 2019

\section{References}

Crowley G (1996) Critical review of ionospheric patches and blobs, in Review of Radio Science 1993-1996. Oxford University Press, New York, p 619

Hosokawa K, Shiokawa K, Otsuka Y, Nakajima A, Ogawa T, Kelly JD (2006) Estimating drift velocity of polar cap patches with all-sky airglow imager at Resolute Bay, Canada. Geophys Res Lett. https://doi.org/10.1029/2006G L026916

Hosokawa K, Shiokawa K, Otsuka Y, Ogawa T, St-Maurice J-P, Sofko GJ, Andre DA (2009a) The relationship between polar cap patches and field-aligned irregularities as observed with an all-sky airglow imager at Resolute Bay and the PolarDARN radar at Rankin Inlet. J Geophys Res. https://doi. org/10.1029/2008JA013707

Hosokawa K, Kashimoto T, Suzuki S, Shiokawa K, Otsuka Y, Ogawa T (2009b) Motion of polar cap patches: a statistical study with all-sky airglow imager at Resolute Bay, Canada. J Geophys Res. https://doi.org/10.1029/2008J A014020

Hosokawa K, Taguchi S, Ogawa Y, Aoki T (2013a) Periodicities of polar cap patches. J Geophys Res. https://doi.org/10.1029/2012JA018165

Hosokawa K, Taguchi S, Ogawa Y, Sakai I (2013b) Two-dimensional direct imaging of structuring of polar cap patches. J Geophys Res. https://doi. org/10.1002/jgra.50577

Hosokawa K, Taguchi S, Shiokawa K, Otsuka Y, Ogawa Y, Nicolls M (2014) Global imaging of polar cap patches with dual airglow imagers. Geophys Res Lett. https://doi.org/10.1002/2013gl058748

Hosokawa K, Taguchi S, Ogawa Y (2016a) Periodic creation of polar cap patches from auroral transients in the cusp. J Geophys Res. https://doi. org/10.1002/2015JA022221

Hosokawa K, Taguchi S, Ogawa Y (2016b) Edge of polar cap patches. J Geophys Res. https://doi.org/10.1002/2015ja021960

Keskinen MJ, Ossakow SL (1983) Theories of high-latitude ionospheric irregularities: a review. Radio Sci. https://doi.org/10.1029/RS018i006p01077

Kubota M, Shiokawa K, Ejiri MK, Otsuka Y, Ogawa T, Sakanoi T, Fukunishi H, Yamamoto M, Fukao S, Saito A (2000) Traveling ionospheric disturbances observed in the OI 630-nm nightglow images over Japan by using a multi-point imager network during the FRONT campaign. Geophys Res Lett 27:4037-4040

Moen J, Gulbrandsen N, Lorentzen DA, Carlson HC (2007) On the MLT distribution of F region polar cap patches at night. Geophys Res Lett 34:L14113. https://doi.org/10.1029/2007GL029632

Ogawa, Y., Y. Tanaka, A. Kadokura, K. Hosokawa, Y. Ebihara, T. Motoba, B. Gustavsson, U. Brandstroem, Y. Sato, S. Oyama, T. Raita, F. Sigernes, S. Nozawa, K. Shiokawa, M. J. Kosch, K. Kauristie, C. Hall, S. Suzuki, Y. Miyoshi, A. Gerrard, H. Miyaoka, and R. Fujii (2019), Development of low-cost multiwavelength imager system for studies of auroras and airglows, submitted to Polar Science 
Otsuka Y, Shiokawa K, Ogawa T, Wilkinson P (2002) Geomagnetic conjugate observations of equatorial airglow depletions. Geophys Res Lett. https:// doi.org/10.1029/2002GL015347

Shiokawa K, Katoh Y, Satoh M, et al. (1999) Development of optical mesosphere thermosphere imagers (OMTI). Earth Planets Space 51:887-896. https://doi.org/10.1186/BF03353247

Shiokawa K, Katoh Y, Satoh M, Ejiri MK, Ogawa T (2000) Integrating-sphere calibration of all-sky cameras for nightglow measurements. Adv Space Res 26(1025-1028):2000

Shiokawa K, Ihara C, Otsuka Y, Ogawa T (2003a) Statistical study of nighttime medium-scale traveling ionospheric disturbances using midlatitude airglow images. J Geophys Res. https://doi.org/10.1029/2002JA009491

Shiokawa K, Otsuka Y, Ihara C, Ogawa T, Rich FJ (2003b) Ground and satellite observations of nighttime medium-scale traveling ionospheric disturbance at midlatitude. J Geophys Res. https://doi.org/10.1029/2002JA0096 39

Shiokawa K, Otsuka Y, Ogawa T (2009) Propagation characteristics of nighttime mesospheric and thermospheric waves observed by optical mesosphere thermosphere imagers at middle and low latitudes. Earth Planets Space 61:479-491. https://doi.org/10.1186/BF03353165
Sobral JHA, Takahashi H, Abdu MA, Muralikrishna P, Sahai Y, Zamlutti CJ, dePaula ER, Batista PP (1993) Determination of the quenching rate of the $\mathrm{O}(1 \mathrm{D})$ by $\mathrm{O}(3 \mathrm{P})$ from rocket-borne optical $(630 \mathrm{~nm})$ and electron density data. J Geophys Res 98:7791-7798. https://doi.org/10.1029/92JA01839

Taguchi M, Okano S, Sakanoi T, Koizumi N, Aso T, Ejiri M (2002) A new meridian imaging spectrograph for the auroral spectroscopy. Adv Polar Upper Atmos Res 16:99-110

Taguchi M, Ejiri M, Tomimatsu K (2004) A new all-sky optics for aurora and airglow imaging. Adv Polar Upper Atmos Res 18:140-148

Taguchi S, Hosokawa K, Ogawa Y, Aoki T, Taguchi M (2012) Double bursts inside a poleward-moving auroral form in the cusp. J Geophys Res. https //doi.org/10.1029/2012JA018150

Weber EJ, Buchau J, Moore JG, Sharber JR, Livingston RC, Winningham JD, Reinisch BW (1984) F layer ionization patches in the polar caps. J Geophys Res 89:1683

\section{Publisher's Note}

Springer Nature remains neutral with regard to jurisdictional claims in published maps and institutional affiliations.

\section{Submit your manuscript to a SpringerOpen ${ }^{\circ}$ journal and benefit from:}

- Convenient online submission

- Rigorous peer review

- Open access: articles freely available online

- High visibility within the field

- Retaining the copyright to your article

Submit your next manuscript at $\gg$ springeropen.com 